University of Nebraska - Lincoln

DigitalCommons@University of Nebraska - Lincoln Infrared Spectroscopy

James E. Baker

USDA-ARS

Floyd E. Dowell

USDA-ARS

James E. Throne

USDA-ARS, Manhattan, KS, james.throne@ars.usda.gov

Follow this and additional works at: https://digitalcommons.unl.edu/usdaarsfacpub

Baker, James E.; Dowell, Floyd E.; and Throne, James E., "Detection of Parasitized Rice Weevils in Wheat Kernels with Near-Infrared Spectroscopy" (1999). Publications from USDA-ARS / UNL Faculty. 1984. https://digitalcommons.unl.edu/usdaarsfacpub/1984

This Article is brought to you for free and open access by the U.S. Department of Agriculture: Agricultural Research Service, Lincoln, Nebraska at DigitalCommons@University of Nebraska - Lincoln. It has been accepted for inclusion in Publications from USDA-ARS / UNL Faculty by an authorized administrator of DigitalCommons@University of Nebraska - Lincoln. 


\title{
Detection of Parasitized Rice Weevils in Wheat Kernels with Near-Infrared Spectroscopy ${ }^{1}$
}

\author{
J ames E. Baker, Floyd E. Dowell, and J ames E. Throne \\ USDA-ARS, Grain Marketing and Production Research Center, 1515 CollegeAvenue, Manhattan, Kansas 66502
}

Received November 24, 1998; accepted March 22, 1999

\begin{abstract}
Individual kernels of wheat containing immature rice weevils, Sitophil us oryzae (L.) (Coleoptera: Curculionidae), parasitized by Anisopteromalus calandrae (Howard) (Hymenoptera: Pteromalidae) could be separated from uninfested kernels and kernels that contained unparasitized weevils by using near-infrared (NIR) spectroscopy. Wheat kernels from cultures of the parasitoid were X-rayed and sorted into uninfested kernels, kernels infested with weevil larvae or pupae, kernels containing parasitoid larvae feeding on host weevils, and kernels containing parasitoid pupae and remains of the host. Kernels were automatically positioned and scanned $(400-1700 \mathrm{~nm})$ with the NIR system. Kernels containing parasitoid pupae were completely differentiated from kernels containing weevil pupae as well as all other kernels in this automated system. With the appropriate integration of NIR spectroscopy and seed-sorting instrumentation, kernels containing parasitoid pupae could be automatically collected from cultures of A. calandrae during the mass rearing of this beneficial insect.

Key Words: biological control; stored products; wheat; parasitoid; Hymenoptera; rice weevil; mass rearing; NIR; near-infrared; detection.
\end{abstract}

\section{INTRODUCTION}

A complex of parasitoids and predators is associated with the stored-grain ecosystem (Brower, 1990). These beneficial insects exert some level of natural control on most stored-grain pest insect populations. However, the best use of these natural enemies in pest management programs in grain stores is through augmentative release (Brower et al., 1996). Optimal release strategies have not been determined for most of the relevant parasitoids. Small cardboard cards containing parasitized eggs have been used for release of Tricho-

\footnotetext{
${ }^{1}$ This article reports results of research only. Mention of a proprietary product does not constitute an endorsement or a recommendation by the USDA for its use.
}

gramma spp. (Schöller et al., 1997). However, in most large-scale pilot tests that have been conducted in stores of whole grain, adult parasitoids have been released directly into storage facilities (Flinn et al., 1996). This is particularly true for parasitoids of internal feeders, such as weevils and borers, for which no method currently exists to differentiate parasitized from unparasitized hosts within grain kernels. Such a method could be advantageous in optimizing release mechanisms with regard to the age and stage of the released parasitoid. For example, dispersal of immature parasitoids could befacilitated by addition of grain kernels containing parasitized hosts during bin loading.

The major parasitoids of in-kernel insect pests of whole grain are Ani sopteromal us cal andrae (Howard), Lariophagous distinguendus (F oerster), Pteromal us cerealellae (Ashmead), and Choetospila elegans (Westwood). These pteromalids are routinely reared on their hosts inside whole grain kernels. Within a single culture, there are uninfested kernels, kernels infested with the pest insects (hosts), kernels with hosts that have been attacked by the parasitoid but not parasitized, and kernels with parasitized hosts from which the adult parasitoid will emerge. If grain kernels containing parasitized hosts are to be used as a vehicle to disseminate or release parasites of different developmental stages, an efficient method is needed to ensure that kernels containing unparasitized pest insects are not inadvertently released into the storage. Burks et al. (1999) demonstrated that low temperatures prevented emergence of unparasitized larvae of the rice weevil Sitophilus oryzae (L.) while not affecting emergence of the parasitoid $\mathrm{A}$. calandrae. We have recently demonstrated that near-infrared (NIR) spectroscopy can be used to detect weevil larvae in individual wheat kernels (Dowell et al., 1998). In this study, we demonstrate that the NIR system can also be used to automatically and rapidly differentiate single wheat kernels containing pupae of the rice weevil parasitoid A. calandrae, separating these from uninfested kernels and kernels containing weevil larvae or pupae. 


\section{MATERIALS AND METHODS}

\section{Insects}

Stock cultures of the rice weevil were maintained on hard red winter wheat (13.5\% moisture content) at $27^{\circ} \mathrm{C}$ and $55-60 \% \mathrm{RH}$. The laboratory strain of $\mathrm{A}$. cal andrae used in this study was maintained on immature rice weevils by transferring adult wasps to wheat infested with 21-day-old weevil larvae. The laboratory strain of A. calandrae has been maintained in culture for $>20$ years.

\section{Selection of Kernels for NIR Analysis}

Wheat kernels were selected from different-aged cultures of $A$. calandrae, X-rayed by the procedures of Throne (1994), and classified into the following categories: uninfested kernels, kernels infested with weevil larvae, kernels infested with weevil pupae, kernels containing parasitoid larvae, and kernels containing parasitoid pupae. Each kernel selected for analysis was uniquely identified and NIR spectra collected within 8 $\mathrm{h}$ of the X-ray procedure. Kernels were held for an additional 3 weeks after NIR spectra were obtained to allow all insects to emerge and to confirm the X-ray identification. Two separate trials with kernels obtained from different insect generations were conducted. The total numbers of individual kernels analyzed in each category were: uninfested kernels $(n=39)$, kernels infested with weevil larvae $(n=28)$, kernels infested with weevil pupae $(n=37)$, kernels containing parasitoid larvae $(n=63)$, and kernels containing parasitoid pupae $(n=40)$.

\section{Kernel Analysis with theNIR System}

A singlekernel characterization system coupled with a diode-array NIR spectrometer (Perten Instruments, Springfield, IL) allowed automated kernel handling and collection of spectra. The system measures visible and NIR absorbance (400-1700 $\mathrm{nm}$ ) but only the NIR region (700-1700 $\mathrm{nm}$ ) was analyzed by partial least squares (PLS) regression (Martens and Naes, 1989) because no visible wavelength absorbance difference was expected. Ability to accurately classify kernels was determined by using coefficients of determination $\left(r^{2}\right)$, standard errors of cross validation (SECV), and percentage of kernels correctly classified. PLS beta coefficients used in calibrations and difference spectra of parasitized and weevil-infested kernels indicated wavelength regions used for classifications. Dowell et al. (1998) describe the data collection method and analysis procedures.

Two comparisons were made. The first comparison determined if kernels infested with either weevils or parasitized weevils could be differentiated from uninfested kernels. Uninfested kernels and kernels with parasitoid or weevil pupae were used to devel op calibrations. All kernels were used in predictions. A second comparison determined if kernels containing parasitoid larvae or pupae (along with remains of the host) could be separated from unparasitized weevil larvae or pupae. A calibration was devel oped using 4/5 of kernels containing parasitoid or weevil pupae. All kernels were used in predictions. Kernels from each trial were analyzed on different dates but all data were combined for analysis.

\section{RESULTS AND DISCUSSION}

Initial analyses determined that uninfested kernels could be separated from kernels infested with either weevils or parasitoids (Table 1 ). Results showed that $97.4 \%$ of uninfested kernels, $100 \%$ of kernels with pupae (host or parasitoid), and $90 \%$ of kernels with larvae (host or parasitoid) were correctly identified. We used 13 factors in the PLS analysis to classify uninfested versus infested kernels $\left(n=116, r^{2}=0.90\right.$, $\mathrm{SECV}=0.15)$. The results agree with previous research by Dowell et al. (1998) which showed that wheat kernels containing 3rd or 4th instar rice weevil larvae could be detected by NIR with greater than $95 \%$ confidence. The misclassified infested kernels likely contained small parasitoid or weevil larvae that did not absorb a threshold amount of NIR radiation.

Subsequent analyses determined that kernels infested with weevil larvae or pupae could be differentiated from kernels with $A$. calandrae pupae (number of factors $=12, n=61, r^{2}=0.87, \mathrm{SECV}=0.18$ ). Table 1 shows that $100 \%$ of kernels containing parasitoid pupae, weevil pupae, and weevil larvae were correctly classified. However, only $14 \%$ of the kernels containing parasitoid larvae were correctly identified. If the detection threshold is changed to correctly classify more parasitoid larvae, then $75 \%$ of the kernels containing parasitoid larvae can be detected while correct classifi-

\section{TABLE 1}

Use of NIR Spectroscopy to Classify Hard Red Winter Wheat Kernels into Uninfested, I nfested with I mmature Rice Weevils, or I nfested with I mmature Rice Weevils Parasitized by Ani sopteromalus calandrae

\begin{tabular}{lllllll}
\hline & \multicolumn{2}{c}{ Kernels correctly classified, \% } \\
\cline { 2 - 3 } & \multicolumn{2}{c}{ Parasitoid } & & Weevils (Hosts) & \\
\cline { 2 - 3 } Comparison & Pupae & Larvae & Pupae & Larvae & Uninfested \\
\cline { 2 - 3 } Uninfested vs infested & 100 & 90 & & 100 & 90 & 97.4 \\
Parasitoid vs weevil & 100 & 14 & & 100 & 100 & - \\
Parasitoid vs weevila & 100 & 75 & 100 & 21 & - \\
\hline
\end{tabular}

a Same as previous comparison, except that the detection threshold is changed to correctly classify more parasitoids. 
cation of parasitoid pupae and weevil pupae remains at $100 \%$. However, $79 \%$ of kernels containing only weevil larvae are misclassified with this calibration.

Spectral analysis showed that the greatest differences in NIR spectra between kernels containing weevils or parasitoids occurred in three wavel ength ranges, 950-1030, 1130-1200, and 1300-1400 nm (Fig. 1). Functional groups responsible for absorbance in the 950-1030 region are $\mathrm{H}_{2} \mathrm{O}$ and second overtones of $\mathrm{RNH}_{2}$ groups, whereas the longer wavelength regions correspond to $\mathrm{C}-\mathrm{H}$ overtones and combinations. The $\mathrm{C}-\mathrm{H}$ overtone regions correspond to chitin absorption regions as reported earlier by Ridgway and Chambers (1996) and Dowell et al. (1998). The present data also provide evidence that NIR may be detecting physical or biochemical differences in the composition of chitin or cuticle between the host and parasitoid within the wheat kernels. However, more detailed studies are needed to confirm this observation.

In summary, NIR spectroscopy can be used to differentiate among individual wheat kernels that are uninfested, those infested with weevil larvae or pupae, or those that contain a parasitoid pupa. With our current system, we could not accurately differentiate between kernels containing weevil larvae and those containing weevil larvae being parasitized by A. calandraelarvae. The inability to differentiate larval parasitoids from host larvae may not be a big disadvantage, however, because of the relatively short duration of the larval stage of this ectoparasitoid compared with the much

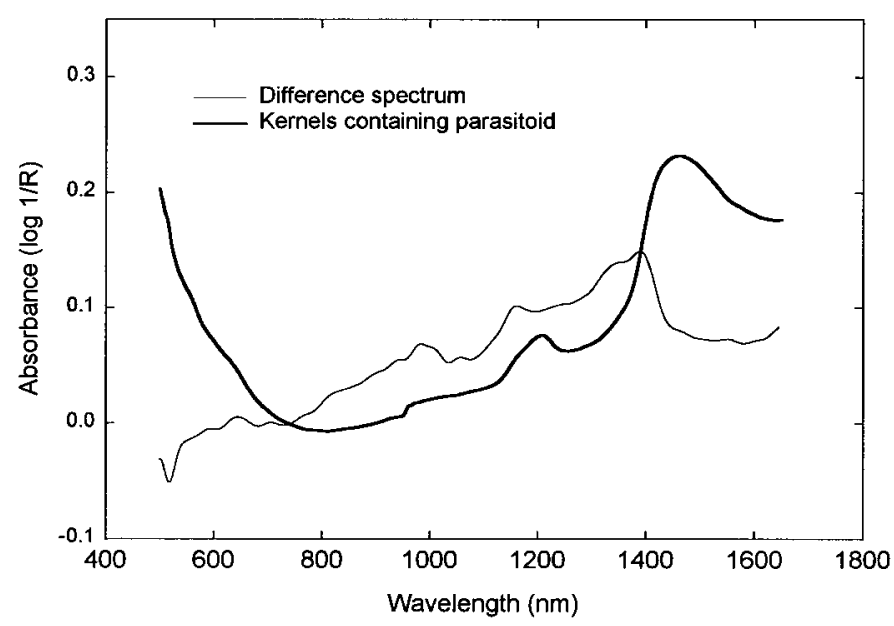

FIG. 1. NIR spectrum of a hard red winter wheat kernel containing a parasitized weevil and the difference spectrum resulting from a spectrum of a kernel containing a parasitized weevil subtracted from a spectrum of a kernel containing an unparasitized weevil. longer duration of the pupal stage (Ghani and Sweetman, 1955).

The NIR system used in this research can scan kernels at a rate of about 2/s. However, technology exists that can sort seeds at speeds to 1000 kernels/s. Adaptation of NIR to this type of instrumentation would provide insectaries that mass rear beneficial insects with a rapid means of collecting large numbers of immature parasitoids of the same stage for shipping and for use in augmentative release programs. The unique characteristics of NIR could also be used for quality control by determining percentage parasitization within mass cultures and to stage classify cultures to ensure synchronous or asynchronous release patterns.

\section{ACKN O WLEDGMENTS}

We thank Ann Redmon and Laurie Peterson for excellent technical assistance

\section{REFERENCES}

Brower, J . H. 1990. Pests of stored products. In "Classical Biological Control in the Southern United States" (D. H. Habeck, F. D. Bennett, and J . H. Frank, Eds.), pp. 113-122. South. Coop. Series, Bulletin No. 355

Brower, J . H., Smith, L., Vail, P. V., and Flinn, P. W. 1996. Biological control. In "I ntegrated Management of I nsects in Stored Products" (B. Subramanyam and D. W. Hagstrum, Eds.), pp. 223-286. Dekker, New York.

Burks, C. S., Hagstrum, D. W., and Baker, J . E. 1999. Selection of cold injury treatments to facilitate release of the parasitoid Anisopteromalus calandrae (Hymenoptera: Pteromalidae) reared on the rice weevil (Coleoptera: Curculionidae). J. Econ. Entomol. 92, 473-479.

Dowell, F. E., Throne, J. E., and Baker, J. E. 1998. Automated nondestructive detection of internal insect infestation of wheat kernels using near-infrared reflectance spectroscopy. J. Econ. Entomol. 91, 899-904.

Flinn, P. W., Hagstrum, D. W., and McGaughey, W. H. 1996. Suppression of beetles in stored wheat by augmentative releases of parasitic wasps. Environ. Entomol. 25, 505-511.

Ghani, M. A., and Sweetman, H. L. 1955. Ecological studies on the granary weevil parasite, Aplastomorpha calandrae (Howard). Biologia 1, 115-139.

Martens, H., and Naes, T. 1989. "Multivariate Calibrations." Wiley, Guildford.

Ridgway, C., and Chambers, J. 1996. Detection of external and internal insect infestation in wheat by near-infrared reflectance spectroscopy. J . Sci. Food Agric. 71, 251-264.

Schöller, M., Prozell, S., Al-Kirshi, A.-G., and Reichmuth, Ch. 1997. Towards biological control as a major component of integrated pest management in stored product protection. J . Stored Prod. Res. 33, 81-97.

Throne, J . E. 1994. Life history of immature maize weevils (Coleoptera: Curculionidae) on corn stored at constant temperatures and relative humidities in the laboratory. Environ. Entomol. 23, 14591471. 